
\title{
25 Research Square \\ Effect of silver diammine fluoride on micro-ecology of plaque from extensive caries of primary teeth - in vitro study
}

Baoying Liu ( $\sim$ smilinglby@163.com )

The First Affiliated Hospital of Zhengzhou University

Jin LIU

The First Affiliated Hospital of Zhengzhou University

Di ZHANG

The First Affilicated Hospital of Zhengzhou University

Zhi lei YANG

The Fist Affiliated Hospital of Zhengzhou University

\section{Ya ping FENG}

The Second Affiliated Hospital of Pingdingshan College

\section{Meng WANG}

The Second Affiliated Hospital of Pingdingshan College

\section{Research article}

Keywords: silver diammine fluoride, plaque micro-ecology, deciduous tooth, dental caries, school children

Posted Date: March 25th, 2020

DOl: https://doi.org/10.21203/rs.3.rs-19319/v1

License: (9) (i) This work is licensed under a Creative Commons Attribution 4.0 International License. Read Full License

Version of Record: A version of this preprint was published at BMC Oral Health on May 24th, 2020. See the published version at https://doi.org/10.1186/s12903-020-01141-3. 


\section{Abstract}

Background The mechanism of action of silver diammine fluoride (SDF) on plaque micro-ecology is seldom studied. This study investigated micro-ecological changes in dental plaque on extensive caries of deciduous teeth after topical SDF treatment. Methods Deciduous teeth with extensive caries freshly removed from school children were collected in clinic. Unstimulated saliva collection and initial plaque sampling were done before tooth extraction, then each caries was topically treated with $38 \%$ SDF in vitro. After intervention, each tooth was stored respectively in artificial saliva at $37^{\circ} \mathrm{C}$. Repeated plaque collections were done at 24 hours and 1 week post-intervention. Post-intervention micro-ecological changes including microbial diversity, microbial metabolism function as well as species correlations were analyzed and compared after pyrosequencing of the DNA from the plaque sample using Illumina MiSeq platform. Results After SDF application, microbial diversity decreased ( $>0.05)$, although not statistically significant. Microbial community composition post-intervention was noticeably different from that of supragingival and pre-intervention plaque as well as saliva. At 1 week post-intervention, the relative content of Pseudomonas , Fusobacterium and Pseudoramibacter was noticeably higher than before, while most of the other bacteria was noticeably reduced, although not statistically significant $(P>0.05)$. The inter-microbial associations became more complex, much more positive associations among survived bacteria were observed than negative ones. COG function classification diagram showed carbohydrate transportation and metabolic functions in the plaque were significantly reduced at 24 hours and 1 week post-intervention. Conclusions SDF has extensive antimicrobial effect on dental plaque, which may reduce carbohydrate metabolism in dental plaque and help promote new balance of the plaque flora.

\section{Background}

Untreated deciduous caries affect 573 million children worldwide[1-3], jeopardize their health and increases the burden of medical care[4]. The incidence of permanent tooth decay increases with the severity of deciduous tooth decay[5]. Fluoride is an important effective weapon in fighting dental caries $[6,7]$, silver diammine fluoride (SDF) garnered great attention because its super ability in arresting and preventing caries [8-13]. It has been called the 'silver bullet' for dental caries [14].

Previous studies on the working mechanism of SDF in the control of dental caries focused mainly on a few specific cariogenic bacteria [15-20], whereas according to the concept of 'ecological plaque hypothesis' [21-24], caries development is due to the loss of micro-ecology balance within the dental plaque. New evidence suggests that SDF has no specific selectivity in inhibiting bacteria [25]. The effect of SDF on micro-ecology of dental plaque should be further investigated.

The aim of this study was to explore the effect of SDF on micro-ecology of carious dental biofilm.

\section{Materials And Methods}


An in vitro study using clinic samples from school children was designed. Ethic approval was gain from the ethics committee of The First Affiliated Hospital of Zhengzhou University (NO. 2018-ky-35). Informed consent from parents or guardians were obtained prior to any clinical examination or sampling.

\section{Subjects}

Children aged 6-12 years admitted to Department of Pediatric Dentistry and Department of Oral and Maxillofacial Surgery in The Second Affiliated Hospital of Pingdingshan College in Henan province, during the period from November 2018 to January 2019 were targeted. Children were examined in the clinic and those with at least one deciduous tooth with extensive untreated caries were recruited.

Inclusion criteria: (1) children aged 6-12 years; (2) the presence of at least one deciduous tooth with extensive caries in the mouth, which needs to be extracted. Exclusion criteria: (1) the extensive caries was restored; (2) the surface of the caries was smooth and hard; (3) children with hereditary or systemic diseases; (4) children with a long history of medication or who have taken antibiotics within the last three months; (5) children who received topical fluoride therapy within the last two weeks.

\section{Collection of plaque and saliva before SDF intervention}

Dental plaque on the tooth surface within the extensive untreated caries (PBI, Plaque Before Intervention), supragingival plaque on smooth surface of permanent first molars (PCF, Plaque from Caries-Free surface) and unstimulated saliva (S, Saliva) were collected from recruited children before tooth extraction. Sterilized dental excavators and sterilized scalers were used for collection of dental plaque within the caries and on sound surfaces, respectively. The collected plaque was put into a $1.5 \mathrm{ml}$ centrifuge tube containing $1 \mathrm{ml}$ PBS solution. Saliva in the amount of $2.5-3 \mathrm{ml}$ was collected using the spitting method described by Fang Yang et al [26]. All collected samples were immediately stored in a freezer at $-20^{\circ} \mathrm{C}$ before laboratory analysis.

\section{SDF intervention}

After extraction, the tooth was carefully cleaned on a sterile petri dish using sterile water and cotton to avoid blood contamination to the caries. An average amount of $10 \mu$ SDF solution was topically applied onto dental surface within the extensive untreated caries by using a pipet, and avoiding touching the surface. A 38\% SDF solution was used in this study, which was prepared in the laboratory of Zhengzhou University according to the method described previously [27]. Briefly, dissolve $12.5 \mathrm{~g} \mathrm{NH}_{4} \mathrm{~F}$ into a volume of distilled water in a $250 \mathrm{ml}$ flask, add $28 \mathrm{~g} \mathrm{Ag}_{2} \mathrm{O}$ powder gradually to the solution, drop $30 \mathrm{ml}$ ammonia $\left(26 \% \mathrm{NH}_{3}\right)$ to the solution and shake the flask continuously to promote reaction in the solution, cool the reaction use cold water during the process, filter the above mix, add distilled water to the filtered solution till the total amount of distilled water added achieve $100 \mathrm{ml}$ and mix the solution well. The ready form of this custom-made SDF solution can receive a $\mathrm{pH}$ value within the range of 8.8-9.4. The tooth was tilted around and assisted with a gentle blow to spread the solution as uniformly as possible on the carious surface. Briefly, the tooth was left undisturbed for 5 minutes before fully submerging into fresh $10 \mathrm{ml}$ 
reduced sterile artificial saliva (Dongguan Yunfei Automation Equipment Technology Co., Ltd., Guangdong, China) separately each in a sterile tube $(50 \mathrm{ml})$ and stored in incubator at $37^{\circ} \mathrm{C}$, ambient air was there surround the tubes. Artificial saliva contains $\mathrm{NaCl} 0.4 \mathrm{~g}, \mathrm{KCl} 0.4 \mathrm{~g}, \mathrm{CaCl}_{2} \cdot 2 \mathrm{H}_{2} \mathrm{O} 0.795 \mathrm{~g}$, $\mathrm{NaH}_{2} \mathrm{PO}_{4} \cdot \mathrm{H}_{2} \mathrm{O} 0.69 \mathrm{~g}, \mathrm{Na}_{2} \mathrm{~S} \cdot 9 \mathrm{H}_{2} \mathrm{O} 0.005 \mathrm{~g}, \mathrm{KSCN} 0.3 \mathrm{~g}$, Urea $1 \mathrm{~g}$ in $1000 \mathrm{ml}$ distilled water. The tooth was transferred to fresh artificial saliva every 24 hours and after plaque sampling procedures.

\section{Collection of plaque after SDF intervention}

At the time of 24 hours and 1 week after SDF intervention, dental plaque within the extensive untreated caries (PAI_24h, Plaque from caries 24 hours After SDF Intervention; PAI_1w, Plaque from caries 1 week After SDF Intervention) was collected and stored again using the same method as mentioned above.

\section{Laboratory processing and analysis}

Samples were sent out to Shanghai Majorbio Bio-pharm Technology Co. Ltd., China for laboratory procedures including DNA extraction, PCR amplification, 16S rDNA sequencing using the lllumina MiSeq platform and sequencing data processing. Laboratory processing was conducted according to standard operating procedures[28]. To ensure the quality of the samples, according to the PCR amplification results provided by this company, samples of quality grade ' $C$ ' will be discarded.

\section{Statistical analysis}

Sequenced dilution curves, alpha diversity, and microbial species counts were calculated and analyzed on the free Majorbio online platform (www.majorbio.com). Bar plot and PCoA diagram were used where appropriate for other qualitative data. PCoA diagram can be obtained from principal co-ordinates analysis (PCOA). PCoA is a method to visual display the microbial similarity of different samples, using mathematic and statistical models to manipulate abundant numbers of sequencing information to summarize and sequence factors which can differentiate microbial diversity of different samples, find the two most important main factors to serve as the horizontal and vertical coordinate, by plotting the diversity characteristics of different samples in this coordinate system, it can not only visualize the microbial diversity, but also display the qualitative data in a quantitative manner, the values on the coordinate lines can represent the relative degree of the difference from point to point. $t$ test was used for pairwise comparison of sample alpha diversity between groups, and the Kruskal-Wallis rank sum test was used for comparison of sample species composition. Network analysis was used to analyze the relationship between bacteria genera. Network analysis can use various mathematic models such as similarity calculation, regression or Bayesian network analysis to build association network of a set of data. In microbial studies, analyzing high throughput sequencing data using mathematic similarity calculation based on pearson or spearman correlation coefficient is usually used to visual display the association network among different Operational Taxonomic Units (OTU) within the microbial sample. In the network diagram, each node represents one OTU, the size of the node represents the OTU abundance, the color of the node is set by the Majorbio online platform according to the advanced taxonomy level to which the OTU belongs. A line between two nodes implies a correlation, the red line is a positive 
correlation, the green line is a negative correlation, the more the lines, the closer the species is to other species. PICRUSt package (Phylogenetic Investigation of Communities by Reconstruction of Unobserved States) was used to analyze the functional characteristics of microorganisms in each group. PICRUSt is a bioinformatics software package freely available from http://picrust.github.io/picrust/, it can predict metagenome functional content from 16S rRNA gene sequencing data. Original version of PICRUSt was used in this study. The statistically significant level was set at $p<0.05$.

\section{Results}

In total, five children were recruited, general demographic and oral health status information of them are shown in Table 1. Each donated one extracted deciduous molar with an extensive untreated caries. A total of 15 plaque samples from carious cavities, 5 saliva samples and 5 supragingival plaque samples were collected. No sample presented a quality grade of ' $C$ ' as shown by PCR amplification results, therefore, all samples passed qualification test for further DNA sequencing.

Table 1 Gender, age and oral health status of the five children in this study

\begin{tabular}{llllll}
\hline Subjects & Gender & Age & Number of permanent / primary tooth & DMFT & dmft \\
\hline NO.1 & female & 8 & $10 / 14$ & 4 & 13 \\
NO.2 & male & 8 & $12 / 10$ & 3 & 10 \\
NO.3 & female & 8 & $12 / 12$ & 0 & 5 \\
NO.4 & male & 9 & $14 / 10$ & 0 & 6 \\
NO.5 & male & 10 & $16 / 8$ & 2 & 8 \\
\hline
\end{tabular}

\section{DNA sequencing information}

A total of 1,280,788 effective sequences were detected (an average of 51232 sequences per sample), with an average sequence length of 427 bp. The dilution curve (Fig. 1) tends to be flat, indicating reasonable and representative sequencing data.

\section{Alpha diversity}

As shown in Fig. 2, in general, microbial diversity of plaque samples from caries as indicated by Shannon indexes decreased over time after the application of SDF, but no statistically significant difference was found $(P>0.05)$. The microbial diversity of PAl-1w were significantly lower than that of saliva samples $(0.01<P<0.05)$.

\section{Microbial community composition and similarity analysis}

The microbial community composition of each group of samples was shown in the bar chart (Fig. 3a). The microbial community composition of treated PAI-24h and PAI-1w samples were noticeably different from that of the untreated PBI group as well as those of saliva and supragingival PCF samples. 
Result of PCoA analysis is shown in Fig. 3b, different degrees of overlap (similarity) among the PBI, PAl24h and PAl-1w samples, and a small overlap between PCF and saliva samples; however, there was no overlap between the former three and the latter two group of samples. The longer the time after intervention, the smaller the overlap among the samples.

The results of community composition diversity test at the genus level is shown in Fig. 4. The results show that the relative contents of Pseudomonas, Fusobacterium and Pseudoramibacter were noticeably higher in PAI-1w samples compared to PBI samples, indicating they are tolerant to SDF intervention, but no statistically significant difference was found $(P>0.05)$. After SDF intervention, the genus of Olsenella, Streptococcus, Bifidobacterium, Prevotella_7, Lactobacillus, Actinomyces, Leptotrichia, Selenomonas_3 and Veillonella showed decreasing trend in different degrees, indicating their sensitivity to SDF intervention, but no statistically significant difference was found $(P>0.05)$.

\section{Network analysis and function prediction}

Fig. 5 is a network diagram made at the genus level, different colors belong to a different phylum. It showed that after SDF intervention, the intergeneric connections became more complex and tight, especially at 1 week after intervention. The positive connections, which indicating collaboration relationships, between genera in the plaque sample after SDF intervention increased compared with that of plaque before intervention, and much more positive associations than negative ones (indicating antagonism relationships) among survived genera was observed at each time point.

Analysis of the microbial functional characteristics based on the COG (cluster of Orthologous Groups) database was carried out (Fig. 6). It showed that the carbohydrate transportation and metabolic functions (shown in yellow in Fig. $6, G)$ were significantly $(p<0.05)$ decreased 1 day and 1 week after SDF intervention. The values of relevant functional parameters for PBI, PAI-24h and PAI- $1 \mathrm{w}$ samples were $0.080,0.068$ and 0.064 , respectively. The value for saliva and PCF samples were 0.067 and 0.066 , respectively. The replication as well as recombination and repair functions (shown in pink in Fig. 6, L) were also significantly reduced, and the signal transduction mechanism (shown in gray in Fig. 6, T) was significantly increased.

\section{Discussion}

Antimicrobial effect of SDF has been proven by various previous studies [15-20,25], but the effect of SDF on micro-ecology of dental plaque has seldom been explored. Based on the already known antimicrobial effect of SDF, this pioneer preliminary study aimed to further our knowledge of SDF on dental plaque from the micro-ecosystem aspect.

In this study, extracted deciduous teeth with large caries from school-age children were collected and topically treated in vitro with a $38 \%$ SDF solution. Dental plaque from the caries pre- and post-intervention were collected for microbial sequencing and the results were compared. The amount of 9 out of 15 richest bacteria genera, the microbial diversity and the microbial community composition in the dental 
plaque after SDF intervention were noticeably changed. The relative proportions of Pseudomonas, Fusobacterium and Pseudoramibacter were higher than before the intervention, although no significant different was found $(P>0.05)$. After SDF intervention, microbial association in the dental plaque became more complex with positive connections overwhelming the negative ones. Carbohydrate transportation and metabolic functions in the dental plaque were significantly reduced $(P<0.05)$.

The microbial diversity was reduced, the microbial richness as well as microbial community composition was shifted post $38 \%$ SDF intervention, which supported the broad spectrum bactericidal effect as shown in previous studies [25], although no statistically significant difference was found in this study. A recent study on $38 \%$ SDF, which compared pre- and one-month-post-intervention dental plaque sample from the mouth of adult patients [29], found no significant difference in the plaque microorganisms composition either. However, difference in dentition, sampling conditions and the follow-up interval should also be noted. The statistical indifference in this study may also be due to the relatively small sample size adopted in this study. Based on experiences of previous studies, a large difference in the microbial diversity before and after SDF intervention was estimated, yielding a number of five children needed in this study. And this relatively small sample size was also practical if balancing the research expenses. Clearly, a larger sample with more funding should be adopted in future studies.

Most of the genera present before the intervention (Olsenella, Streptococcus, Bifidobacterium, Prevotella_7, Lactobacillus, Actinomyces, Leptotrichia, Selenomonas_3, Veillonella) were sensitive to SDF, and the relative amounts decreased substantially. This was consistent with previous studies $[15,16,18$, 20] and supported the broad spectrum bactericidal effect of SDF [25]. Streptococcus, Lactobacillus and Actinomyces have long been recognized as classical cariogenic bacteria. Prevotella_7, Selenomonas_3, and Bifidobacterium are often found in deep dentin caries [30]. Olsenella, which could produce lactic acid, seldom mentioned in the past, have recently been found associated with dentin and root caries [31, 32]. Leptotrichia has a strong glycolysis effect [33], and its detection rate is increased in the process of caries $[34,35]$. Veillonella has a unique intracellular pH control mechanism [36], which might promote the cariogenicity of Streptococcus mutans [35,37]. By inhibiting these bacteria, cariogenicity of the dental plaque may be suppressed, creating a new microbial balance.

It was also found that Pseudomonas, Fusobacterium and Pseudoramibacter could withstand the effect of topically applied SDF. The possible role of Pseudomonas in dental caries was indicated recently. NavNeet Kaur et al. [38] detected a high level of Pseudomonas in the dentin caries. In Liang's study [39], Pseudomonas was mentioned as a potential pathogen of caries, but suggested the relationship be further studied. Pseudomonas is a non-fermenting, obligate aerobic Gram-negative bacillus. It has no special nutritional requirements and is common in soil, fresh water, seawater. A total of twenty-nine species have been identified, of which at least three are pathogenic to animals or humans, causing infections. Pseudomonas aeruginosa is part of the commensal oral flora, but can become a pathogen and cause nosocomial infection [40]. Small infective doses of the bacteria can produce local abscesses, large doses can lead to death from systemic infection. Fluorescent pseudomonas ssp. can cause spoilage of frozen meat, eggs, milk and dairy products. Pseudomonas birensis can be transmitted through the mouth, the 
respiratory tract or in wounds. Pseudoramibacter is a nonmotile, nonsporeforming, strictly anaerobic, Gram-positive bacillus, which was saccharolytic. The end products of its fermentation are formate, acetate, butyrate, caproate, and hydrogen [41]. It can cause periapical infection [42-44], it often found in deep dentin caries [30,45] and infected root canals [41]. Similarly, Fusobacterium is often detected in deep dentin caries $[30,45]$ and infected root canals $[43,44,46]$. It is a strictly anaerobic Gram-negative bacillus living in the oral cavity and the upper digestive, intestine and urogenital tracts of humans or animals, as well as in the soil. It is most commonly seen in oral dental plaque. Most of the strains do not ferment any sugars, only a few strains are known to weakly ferment glucose and fructose. Briefly, Pseudomonas, Fusobacterium and Pseudoramibacter may not play important role in the development of dental caries, but they are more closely related to root canal or periapical infections. In this study, deciduous teeth with extensive caries or obvious dental pulp symptoms from school-age children was studied, which could explain the richness of these bacteria in the sample. The results would also provide guiding information in the prevention and control of extensive deciduous caries associated with pulp infection or periapical disease. The tolerance of these three kind of bacteria to single time SDF application in this in vitro study should be pay cautious attention to when use SDF in the clinical situation in child population. Further studies are worth conducting and should be designed according to this special concern in order to form a clear guide.

Restricted by ethic issues, extracted deciduous teeth were used in this study. As a result, intervention and sampling were all carried out in vitro. An in vitro study differs from the real clinical situation. The effect of common oral flora, saliva, food and environmental microorganisms on the formation of new microbial balance could not be reproduced or evaluated. New micro-ecological balance formation after SDF intervention in this study could only be based on the before-intervention status as well as the effect of SDF. Bacteria which were sensitive to SDF were controlled, while those with relatively high tolerance to SDF grew relatively better after the intervention. After SDF intervention, the microbial association in the dental plaque became more complex with positive connections overwhelming the negative ones, indicating that the connections and collaborations among the remaining bacterial communities were enhanced.

The functional characteristics of plaque in caries were also compared and analyzed based on the COG database for homologous classification of gene products. Carbohydrate transportation and metabolism function in plaque were significantly reduced 24 hours and 1 week after intervention (shown in yellow in Fig. 6, G), and the replication, recombination and repair function in plaque (shown in pink in Fig. 6, L) were also significantly reduced. These changes revealed from another aspect the overall inhibitory effect of SDF on the bacterial community in dental plaque, which affected the properties of the whole plaque, especially the cariogenicity within the plaque micro-ecology. The function of signal transduction mechanism was significantly increased, which could be correlated with the enhancement of synergistic effect between residual bacteria in plaque after intervention.

Most parameters in this study showed that the greatest microbial difference was found at one week after SDF intervention, indicating a possible time effect of SDF. However, the observation period in this study 
was relatively short which may have its limitations, and there were differences between this in vitro study and the real mouth situation, time effect of SDF application on dental plaque may not be well explained, and should be studied further in good experiment situation which can mimic the real mouth environment well or in real mouth situation if possible.

\section{Conclusions}

Within the limitation of this study, it is concluded that, by focusing on different perspective of dental plaque micro-ecosystem, this study supported the concept of 'ecological plaque hypothesis' in understanding the development of dental caries. Topical application of 38\% SDF had extensive inhibitory effect on dental plaque microorganisms, which led to reduced carbohydrate metabolism and increased positive connections among surviving bacteria. It also provided an opportunity for redistribution of the plaque micro-ecology. This study may promote our understanding of the caries prevention and control mechanism of SDF on dental plaque from the micro-ecology perspective.

\section{Abbreviations}

SDF, silver diammine fluoride.

\section{Declarations}

- Ethics approval and consent to participate

Ethic approval was gain from the ethics committee of The First Affiliated Hospital of Zhengzhou University (NO. 2018-ky-35). Informed consent from parents or guardians were obtained prior to any clinical examination or sampling.

- Consent for publication

Not applicable

- Availability of data and materials

The datasets used and/or analysed during the current study are available from the corresponding author on reasonable request.

- Competing interests

We have no competing interests.

- Funding

This study was supported by the Youth Fund of The First Affiliated Hospital of Zhengzhou University (Code Number YNQN2017064). 
LBY conceptualized and designed the study, guided the conduction of the experiment and was a major contributor in results explanation, drafting and revising the manuscript. LJ guided the conduction of the experiment, contributed in results explanation and drafting of the manuscript. ZD carried out the study and the experiment, conducted data analysis and involved in the drafting of this manuscript. YZL helped the conduction of the study and involved in the data analysis and results interpretation. FYP and WM assisted in the conduction of the study (clinical screening of experimental subjects and the sampling).

\section{- Acknowledgements}

We would like to thank the children and their families for their participation, as well as Shanghai Majorbio Bio-pharm Technology Co. Ltd., China for their kind help with Illumina MiSeq sequencing, and the data were analyzed using the I-Sanger Cloud Platform.

\section{Reference}

1. Shirk C. Oral health checkup: progress in tough fiscal times? Issue Brief George Wash Univ Natl Health Policy Forum. 2010(836):1-18.

2. Kassebaum NJ, Smith AGC, Bernabe E, Fleming TD, Reynolds AE, Vos T, et al. Global, Regional, and National Prevalence, Incidence, and Disability-Adjusted Life Years for Oral Conditions for 195 Countries, 1990-2015: A Systematic Analysis for the Global Burden of Diseases, Injuries, and Risk Factors. J Dent Res. 2017;96(4):380-7.

3. Colak H, Dulgergil CT, Dalli M, Hamidi MM. Early childhood caries update: A review of causes, diagnoses, and treatments. J Nat Sci Biol Med. 2013;4(1):29-38.

4. Rosier BT, Jager MD, Zaura E, Krom BP. Historical and contemporary hypotheses on the development of oral diseases: are we there yet? Frontiers in cellular and infection microbiology. 2014;4.

5. Almeida AG, Roseman MM, Sheff M, Huntington N, Hughes CV. Future caries susceptibility in children with early childhood caries following treatment under general anesthesia. Pediatric dentistry. 2000;22(4):302-6.

6. Marinho VC, Worthington HV, Walsh T, Clarkson JE. Fluoride varnishes for preventing dental caries in children and adolescents. The Cochrane database of systematic reviews. 2013(7):Cd002279.

7. Walsh T, Worthington HV, Glenny AM, Appelbe P, Marinho VC, Shi X. Fluoride toothpastes of different concentrations for preventing dental caries in children and adolescents. The Cochrane database of systematic reviews. 2010(1):Cd007868.

8. Zhao IS, Gao SS, Hiraishi N, Burrow MF, Duangthip D, Mei ML, et al. Mechanisms of silver diammine fluoride on arresting caries: a literature review. International dental journal. 2018;68(2):67-76.

9. $\mathrm{Chu} \mathrm{CH}$, Lo EC. Promoting caries arrest in children with silver diammine fluoride: a review. Oral health \& preventive dentistry. 2008;6(4):315-21. 
10. Horst JA, Ellenikiotis H, Milgrom PL. UCSF Protocol for Caries Arrest Using Silver diammine Fluoride: Rationale, Indications and Consent. J Calif Dent Assoc. 2016;44(1):16-28.

11. Mei ML, Lo EC, Chu CH. Clinical Use of Silver diammine Fluoride in Dental Treatment. Compendium of continuing education in dentistry (Jamesburg, NJ : 1995). 2016;37(2):93-8; quiz100.

12. Mei ML, Chu CH, Lo EC, Samaranayake LP. Fluoride and silver concentrations of silver diammine fluoride solutions for dental use. International journal of paediatric dentistry. 2013;23(4):279-85.

13. Peng JJ, Botelho MG, Matinlinna JP. Silver compounds used in dentistry for caries management: a review. Journal of dentistry. 2012;40(7):531-41.

14. Rosenblatt A, Stamford TC, Niederman R. Silver diammine fluoride: a caries "silver-fluoride bullet". J Dent Res. 2009;88(2):116-25.

15. Chu CH, Mei L, Seneviratne CJ, Lo EC. Effects of silver diammine fluoride on dentine carious lesions induced by Streptococcus mutans and Actinomyces naeslundii biofilms. International journal of paediatric dentistry. 2012;22(1):2-10.

16. Mei ML, Li QL, Chu CH, Lo EC, Samaranayake LP. Antibacterial effects of silver diammine fluoride on multi-species cariogenic biofilm on caries. Ann Clin Microbiol Antimicrob. 2013;12:4.

17. Mei ML, Ito L, Cao Y, Li QL, Lo EC, Chu CH. Inhibitory effect of silver diammine fluoride on dentine demineralisation and collagen degradation. Journal of dentistry. 2013;41(9):809-17.

18. Mei ML, Ito L, Cao Y, Lo EC, Li QL, Chu CH. An ex vivo study of arrested primary teeth caries with silver diammine fluoride therapy. Journal of dentistry. 2014;42(4):395-402.

19. Mei ML, Lo ECM, Chu CH. Arresting Dentine Caries with Silver diammine Fluoride: What's Behind It? 2018;97(7):751-8.

20. Liu BY, Mei L, Chu CH, Lo ECM. Effect of Silver Fluoride in Preventing the Formation of Artificial Dentinal Caries Lesions in vitro. The Chinese journal of dental research : the official journal of the Scientific Section of the Chinese Stomatological Association (CSA). 2019;22(4):273-80.

21. Marsh PD. Microbial ecology of dental plaque and its significance in health and disease. Advances in dental research. 1994;8(2):263-71.

22. Marsh PD. Dental plaque: biological significance of a biofilm and community life-style. Journal of clinical periodontology. 2005;32 Suppl 6:32 Suppl 6.

23. Marsh PD. Dental plaque as a biofilm and a microbial community - implications for health and disease. BMC oral health. 2006;6 Suppl 1:S14.

24. Takahashi N, Nyvad B. The role of bacteria in the caries process: ecological perspectives. J Dent Res. 2011;90(3):294-303.

25. Milgrom P, Horst JA, Ludwig S, Rothen M, Chaffee BW, Lyalina S, et al. Topical silver diammine fluoride for dental caries arrest in preschool children: A randomized controlled trial and microbiological analysis of caries associated microbes and resistance gene expression. Journal of dentistry. 2018;68:72-8. 
26. Yang F, Ning K, Zeng X, Zhou Q, Su X, Yuan X. Characterization of saliva microbiota's functional feature based on metagenomic sequencing. SpringerPlus. 2016;5(1):2098.

27. Peng MW, Li YJ, Li JY, Huang CW, Qiu XQ. Anti-caries agent-Preparation and mensuration of silver diammine fluoride. Chinese Pharmaceutical Journal. 1982(07):15-6.[in Chinese]

28. Jiang Q, Liu J, Chen L, Gan N, Yang D. The Oral Microbiome in the Elderly With Dental Caries and Health. Frontiers in cellular and infection microbiology. 2018;8:442.

29. Mitwalli H, Mourao MDA, Dennison J, Yaman P, Paster BJ, Fontana M. Effect of Silver diammine Fluoride Treatment on Microbial Profiles of Plaque Biofilms from Root/Cervical Caries Lesions. Caries research. 2019;53(5):555-66.

30. Rocas IN, Alves FR, Rachid CT, Lima KC, Assuncao IV, Gomes PN, et al. Microbiome of Deep Dentinal Caries Lesions in Teeth with Symptomatic Irreversible Pulpitis. PLoS One. 2016;11(5):e0154653.

31. Kraatz M, Wallace RJ, Svensson L. Olsenella umbonata sp. nov., a microaerotolerant anaerobic lactic acid bacterium from the sheep rumen and pig jejunum, and emended descriptions of Olsenella, Olsenella uli and Olsenella profusa. Int J Syst Evol Microbiol. 2011;61(Pt 4):795-803.

32. Jiang $W$, Ling $Z$, Lin $X$, Chen $Y$, Zhang J, Yu J, et al. Pyrosequencing analysis of oral microbiota shifting in various caries states in childhood. Microbial ecology. 2014;67(4):962-9.

33. Thompson J, Pikis A. Metabolism of sugars by genetically diverse species of oral Leptotrichia. Molecular oral microbiology. 2012;27(1):34-44.

34. Morou-Bermudez E, Rodriguez S, Bello AS, Dominguez-Bello MG. Urease and Dental Plaque Microbial Profiles in Children. PLoS One. 2015;10(9):e0139315.

35. Richards VP, Alvarez AJ, Luce AR, Bedenbaugh M, Mitchell ML, Burne RA. Microbiomes of SiteSpecific Dental Plaques from Children with Different Caries Status. 2017;85(8).

36. Do T, Sheehy EC, Mulli T, Hughes F, Beighton D. Transcriptomic analysis of three Veillonella spp. present in carious dentine and in the saliva of caries-free individuals. Frontiers in cellular and infection microbiology. 2015;5:25.

37. Wang YX, Zhou XD, Li MY. A review on the role of veillonella in caries and its interaction with Streptococcus. International Journal of Stomatology. 2017;44(2):195-9. [in Chinese]

38. Kaur N, SahNi P, SiNghvi a, haNS MK, ahluwalia aS. Screening the Drug Resistance Property Among Aerobic Pathogenic Microorganisms of Dental Caries in North-Western Indian Population: A Preliminary Study. Journal of Clinical and Diagnostic Research. 2015;9(7):ZC05-ZC8.

39. Xiao C, Ran S, Huang Z, Liang J. Bacterial Diversity and Community Structure of Supragingival Plaques in Adults with Dental Health or Caries Revealed by 16 S Pyrosequencing. Frontiers in microbiology. 2016;7:1145.

40. Senpukua H, Sogameb A, Inoshitac E, Tsuhaa Y, Miyazakid H, Hanada N. Systemic Diseases in Association with Microbial Species in Oral Biofilm from Elderly Requiring Care. Gerontology. 2003;49(5):301-9. 
41. Siqueira JF, Jr., Rocas IN. Pseudoramibacter alactolyticus in primary endodontic infections. Journal of endodontics. 2003;29(11):735-8.

42. Willmann C, Mata X, Hanghoej K, Tonasso L, Tisseyre L, Jeziorski C, et al. Oral health status in historic population: Macroscopic and metagenomic evidence. PLoS One. 2018;13(5):e0196482.

43. Lee LW, Lee YL, Hsiao SH, Lin HP. Bacteria in the apical root canals of teeth with apical periodontitis. Journal of the Formosan Medical Association = Taiwan yi zhi. 2017;116(6):448-56.

44. Tatikonda A, Sudheep N, Biswas KP, Gowtham K, Pujari S, Singh P. Evaluation of Bacteriological Profle in the Apical Root Segment of the Patients with Primary Apical Periodontitis. The Journal of Contemporary Dental Practice. 2017;18(1):44-8.

45. Rocas IN, Lima KC, Assuncao IV, Gomes PN, Bracks IV, Siqueira JF, Jr. Advanced Caries Microbiota in Teeth with Irreversible Pulpitis. Journal of endodontics. 2015;41(9):1450-5.

46. Yun $\mathrm{KH}$, Lee HS, Nam OH. Analysis of bacterial community profiles of endodontically infected primary teeth using pyrosequencing. 2017;27(1):56-65.

\section{Figures}




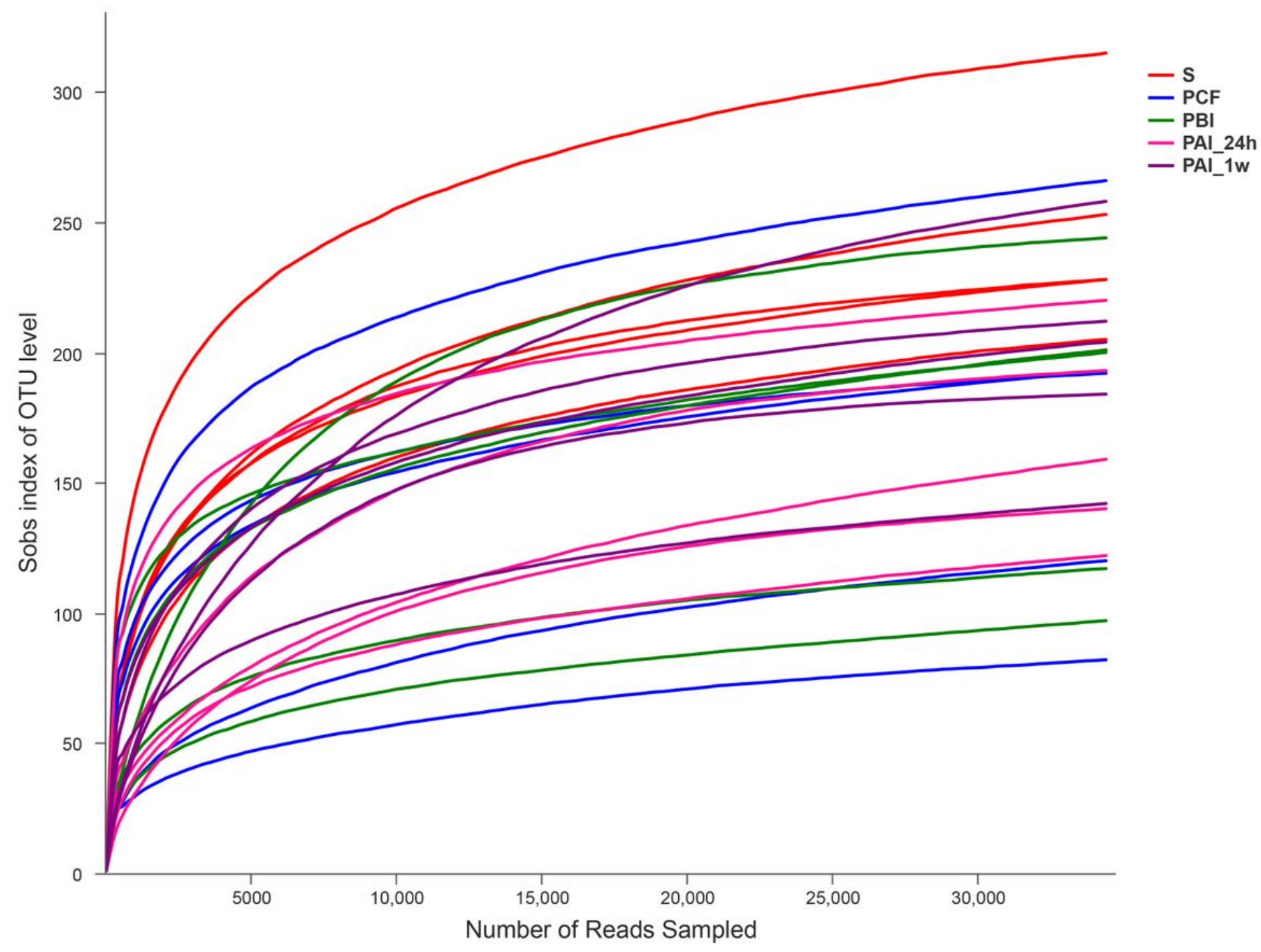

Figure 1

Dilution curves of samples. Each sample is shown separately by a single line. Different color represents different group of samples as indicated in the icons aside. Sobs index refers to the actual observed value of richness, curves were constructed by using the Sobs index of each sample at different sequencing depths. 


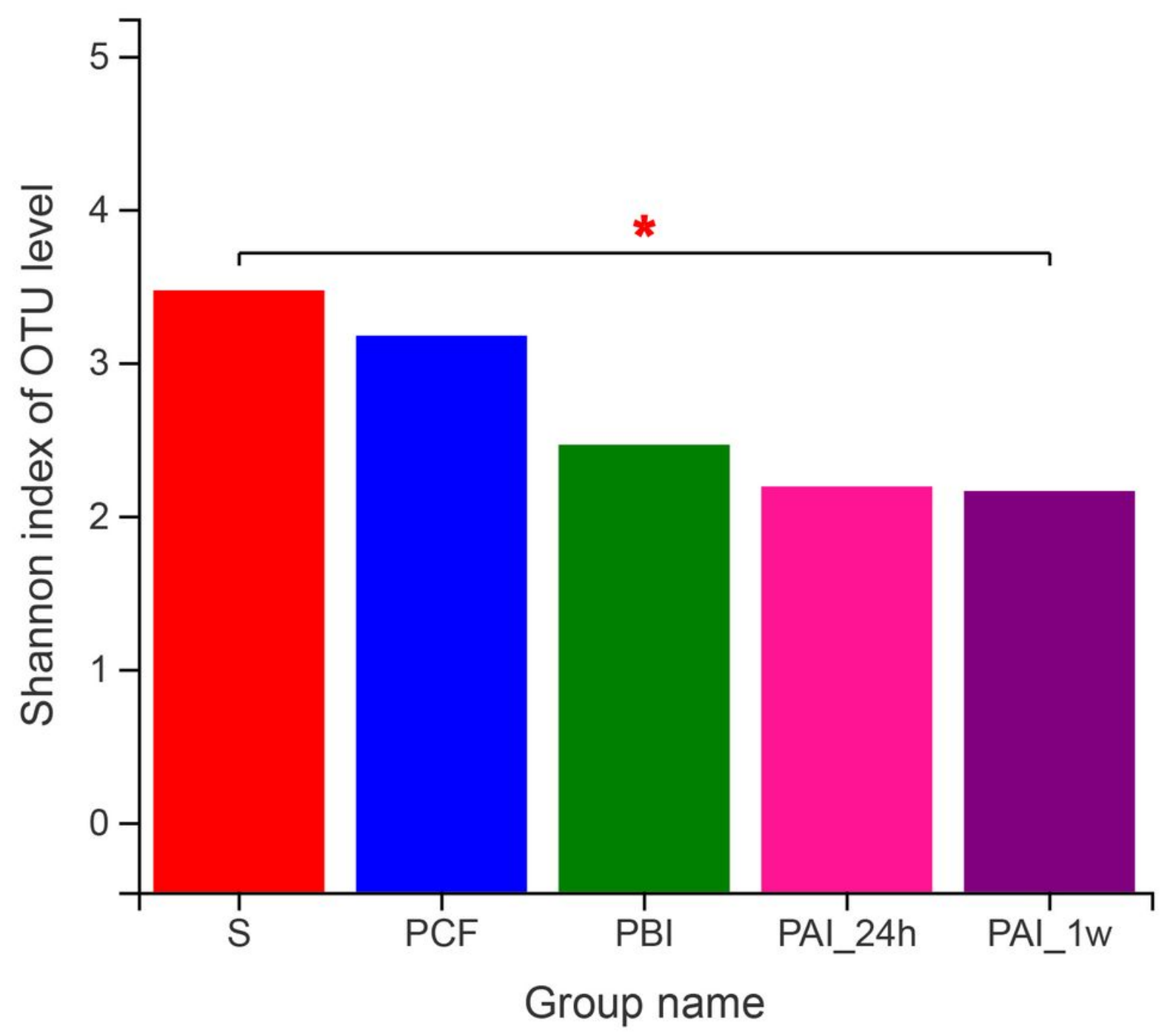

$\square S$

$\square \mathrm{PCF}$

PBI

DAl_24h

aAl_1w

Figure 2

Alpha diversity as measured by the Shannon index. * $0.01<\mathrm{P} \leq 0.05$ 

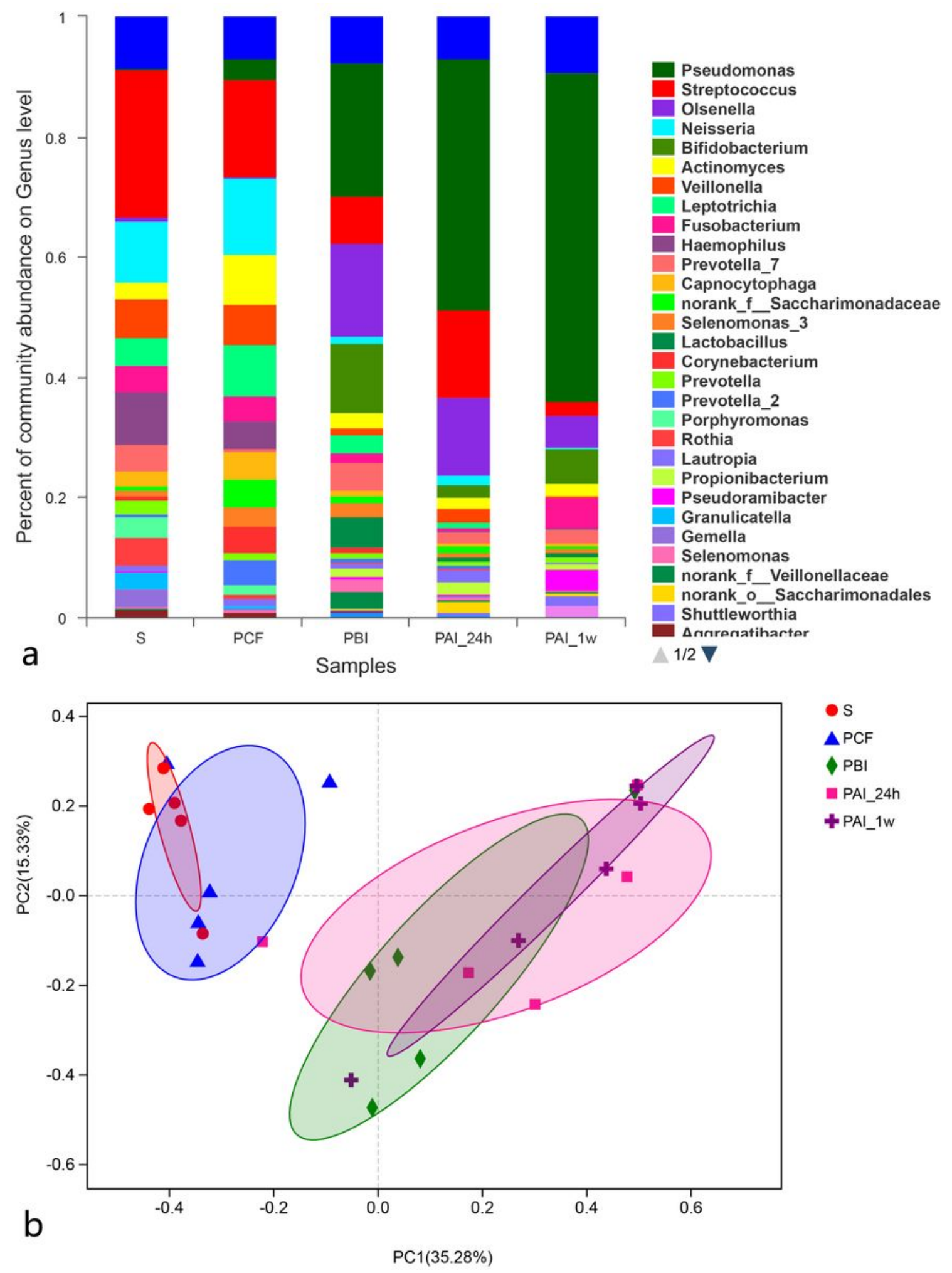

\section{Figure 3}

Community bar plot analysis and PCoA diagram. $\mathrm{a}$ is the Community bar plot, the abscissa is the sample name, and the ordinate is the proportion of species in the sample. $b$ is the PCoA diagram on OTU level. The $\mathrm{X}$-axis and $\mathrm{Y}$-axis represent the two selected primary axes, and the percentage represents the explanatory value of the primary axis to the difference in sample composition, the scale of the X-axis and $\mathrm{Y}$-axis is the relative distance, which is meaningless. Dots of different colors or shapes represent samples 
of different groups. The closer the two sample points are, the more similar is the species composition of the two samples.
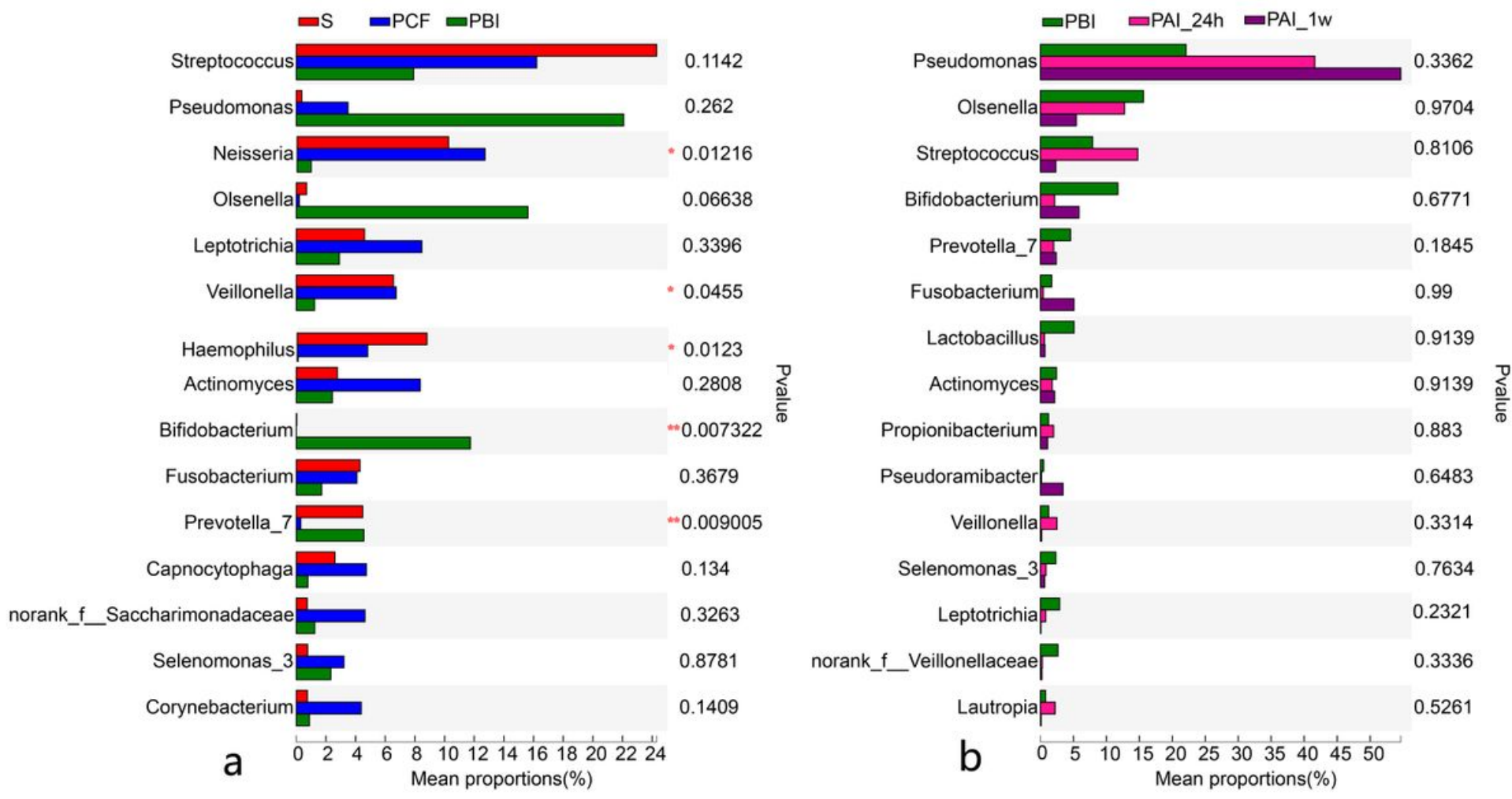

\section{Figure 4}

Community composition diversity test on the top 15 richest genera among different groups of samples. a is for baseline, $b$ is for the application of SDF. ${ }^{*} 0.01<P \leq 0.05 \nabla^{\star \star} 0.001<P \leq 0.01$. 


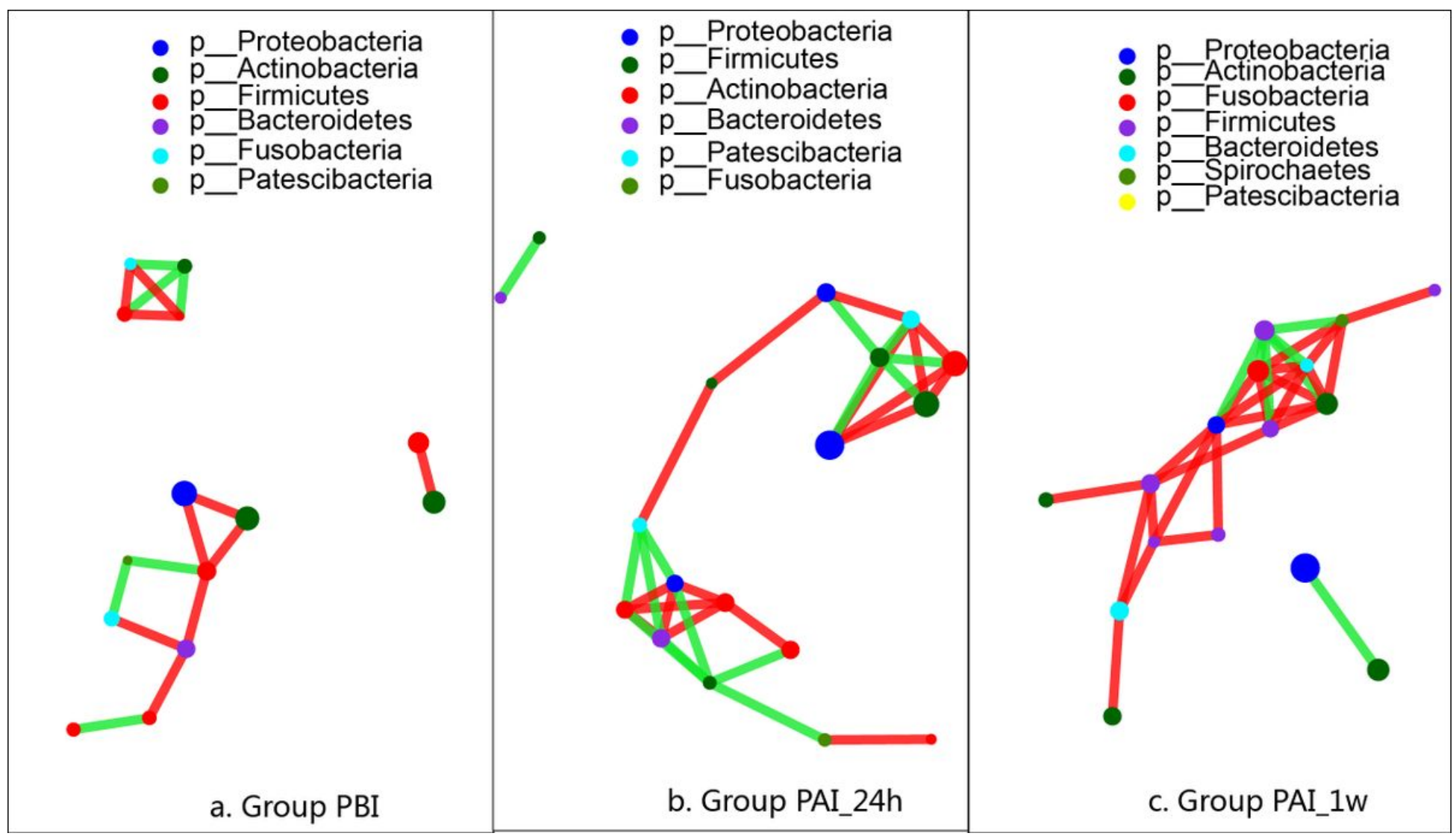

Figure 5

Genus correlation network. The size of the node represents the genus abundance, but different colors of the node represent a different phylum. A line between two nods implies a correlation, red line means positive correlation, green line means negative; the more the lines, the closer the genus is to other genera. 


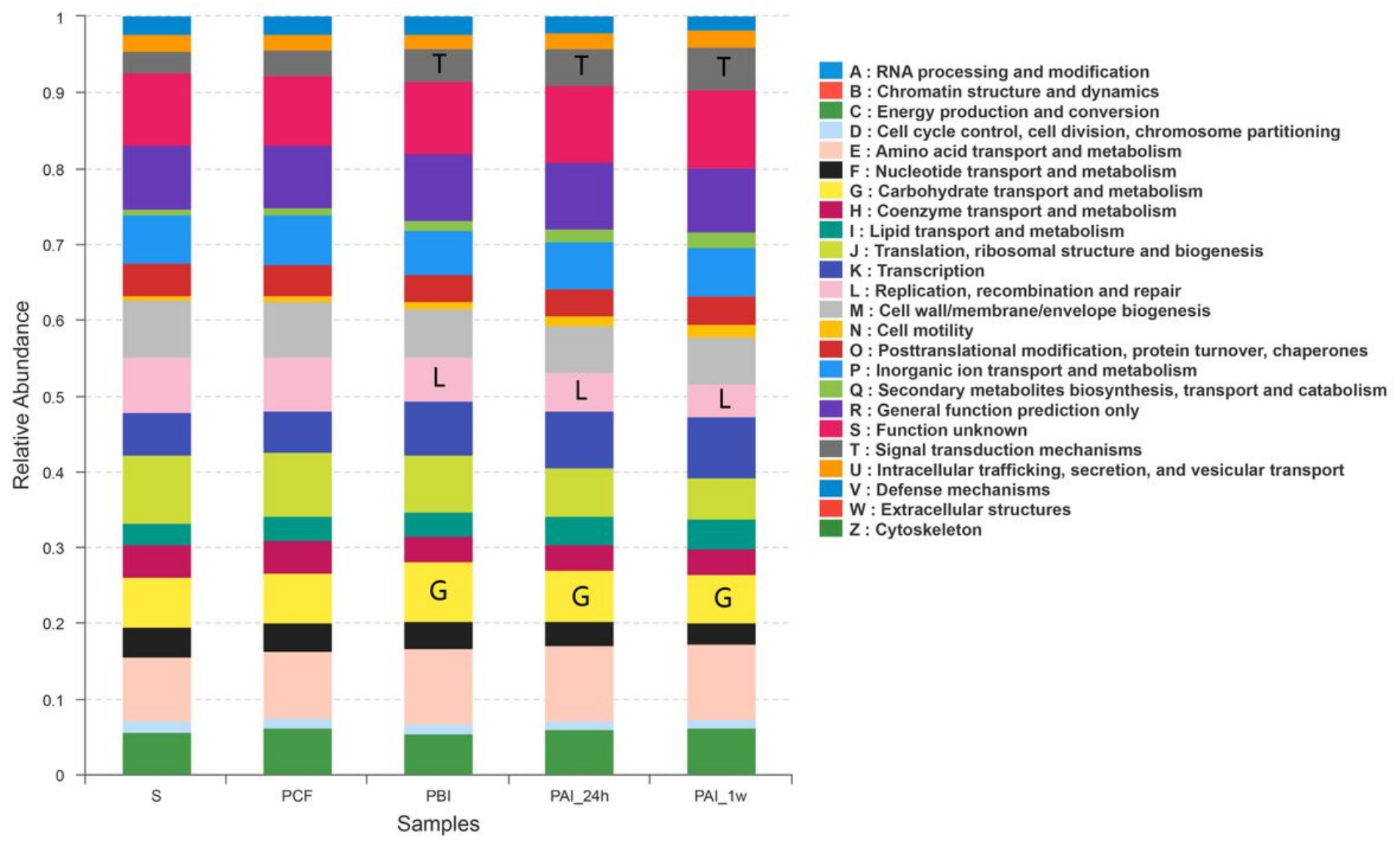

Figure 6

COG function classification. 